\title{
A competitive ligand-binding assay for the detection of neutralizing antibodies against dostarlimab (TSR-042)
}

\author{
Xiaolong Tom Zhang ${ }^{1}$, Hong Chen ${ }^{2}$, Weiping Shao ${ }^{3}$, Zhongping John Lin ${ }^{3}$, Murad Melhem ${ }^{4}$ and Sharon Lu ${ }^{4}$
}

\begin{abstract}
Dostarlimab is a humanized anti-PD-1 monoclonal antibody. Dostarlimab (JEMPERLI;TSR-042) was recently approved in the USA and in the EU. The presence of neutralizing antibodies (NAbs) is a cause for concern because they block the therapeutic function of the antibody and reduce drug efficacy. Therefore, programs developing therapeutic biologics need to develop and validate assays that adequately assess the presence of NAbs in the serum of patients treated with biologic therapies. Presented here is the development and validation of a competitive ligand-binding assay that specifically detects anti-dostarlimab NAbs in human serum. Precision, sensitivity, hook effect, selectivity, assay robustness, stabilities, and system suitability were evaluated. In addition, drug tolerance of the assay with the implementation of a drug removal process was investigated. The cut point factor for the detection of NAbs in human serum at a $1 \%$ false-positive rate was determined. The assay's precision, sensitivity, hook effect, selectivity, robustness, and drug interference were tested and found to be acceptable. With system suitability and stability established, this assay has been used to evaluate NAbs to guide the development of dostarlimab.

Trial registration: Clinicaltrials.gov, NCT02715284. Registered 9 March 2016

Keywords: Dostarlimab, Endometrial cancer, Neutralizing antibodies, Immunotherapy, Competitive ligand-binding assay
\end{abstract}

\section{Introduction}

Immunotherapy with antibodies directed at the programmed death 1 (PD-1)/PD ligand 1(PD-L1) axis has shown remarkable activity in a variety of tumor types in clinical trials and has been approved worldwide for a number of malignancies (Robert et al. 2014; Weber et al. 2015; Fehrenbacher et al. 2016; Antonia et al. 2017; Apolo et al. 2017; Seiwert et al. 2016; Ansell et al. 2015; El-Khoueiry et al. 2017; Kaufman et al. 2016; Gong et al. 2018; Ahn et al. 2019; Khozin et al. 2019). Dostarlimab (JEMPERLI; TSR-042) is a humanized anti-PD-1

\footnotetext{
*Correspondence: slu@scholarrock.com

${ }^{4}$ GlaxoSmithKline, 1000 Winter Street, Waltham, MA 02451, USA Full list of author information is available at the end of the article Weiping Shao was employed by Frontage Laboratories when the research was conducted.
}

monoclonal antibody (mAb) (Oaknin et al. 2018). In the USA, dostarlimab was recently approved as a monotherapy in adult patients with mismatch repair-deficient (dMMR) recurrent or advanced endometrial cancer that has progressed on or after a platinum-containing regimen or dMMR recurrent or advanced solid tumors that have progressed on or following prior treatment and who have no satisfactory alternative treatment options (US Food and Drug Administration 2021a; US Food and Drug Administration 2021b; Andre et al. 2021; ClinicalTrials.gov 2016). In the EU, dostarlimab was recently approved as a monotherapy in adult patients with recurrent or advanced $\mathrm{dMMR} /$ microsatellite instability-high endometrial cancer that has progressed on or after treatment with a platinum-containing regimen (Oaknin et al. 2018; Andre et al. 2021; ClinicalTrials.gov 2016; European Medicines Agency 2021). As with other 
immunotherapeutic agents, anti-drug antibodies (ADAs) may emerge and be detectable after administration of dostarlimab (Pineda et al. 2016). However, dostarlimab is a humanized $\mathrm{mAb}$, and as such, has a lower risk of eliciting ADAs or neutralizing (NAbs) than non-humanized mAbs (Davda et al. 2019).

However, the production of ADAs is generally a treatment risk with therapeutic antibodies and can lead to an altered pharmacokinetic (PK) profile, which in turn may impact the safety and efficacy of therapeutic antibodies (Garcês and Demengeot 2018; Bloem et al. 2017). ADAs can be (1) binding, leading to minimal or no impact; (2) clearing, leading to impact through an altered PK profile; (3) sustaining, leading to a prolonged exposure that may change the efficacy and/or safety; or (4) neutralizing, leading to reduced pharmacological activity of the therapeutic antibody (Garcês and Demengeot 2018; Bloem et al. 2017).

Thus, the presence of NAbs is concerning because they block the therapeutic function of the antibody and are likely to lead to reduced efficacy (Garcês and Demengeot 2018; Bloem et al. 2017; Gunn et al. 2016). Furthermore, NAbs may lead to safety issues, such as hypersensitivity and anaphylaxis Gunn et al. 2016; Krishna and Nadler 2016). Therefore, programs developing therapeutic antibodies need to develop and validate assays that adequately assess the presence of NAbs in the serum of patients treated with such therapeutic agents.

Cell-based reporter assays have been developed and validated for detecting NAbs, including a cell-based bioluminescent reporter assay that quantifies therapeutic mAbs against PD-1 and PD-L1 (Liao et al. 2012; Cong et al. 2015; Jolicoeur and Tacey 2012).

Regulatory agencies prefer cell-based assays because they rely on cellular responses to NAb-mediated inhibition of therapeutic antibodies (Wu et al. 2016). Because of their mode of detection, these assays have been considered more biologically relevant than noncell-based assays (Bloem et al. 2017; Liao et al. 2012; Cong et al. 2015; Jolicoeur and Tacey 2012). However, cell-based assays are labor intensive and highly variable and exhibit low serum tolerance and poor drug tolerance (Bloem et al. 2017; Liao et al. 2012; Cong et al. 2015; Jolicoeur and Tacey 2012; Wu et al. 2016). In contrast to cell-based assays, non-cell-based assays often rely on binding of the drug and target for signal detection and quantitation. Competitive ligand-binding assays, used to characterize NAbs, have been shown to provide higher sensitivity, a wider dynamic range, increased precision, and better matrix tolerance than cell-based assays (Wu et al. 2018). Although regulatory agencies generally prefer cell-based assays, the United States Food and Drug Administration and the European
Medicines Agency recognize both cell-based and noncell-based competitive ligand-binding assays as valid measurements of NAbs in some cases (Jolicoeur and Tacey 2012; Wu et al. 2016). The selection of either cellbased or non-cell-based assays is driven by several different variables, including therapeutic mechanism of action (MOA), assay performance characteristics, and risk of immunogenicity, with therapeutic MOA being the primary determinant (Wu et al. 2016).

Here, we present the development and validation of a competitive ligand-binding assay that is specific for the detection of anti-dostarlimab NAbs in human serum. We validated the assay's precision, sensitivity, hook effect, selectivity, robustness, stability, and system suitability. In addition, we investigated the drug tolerance of the assay with the implementation of a drug removal process, as well as the possibilities for false-negative results. The utility of this assay as it pertains to the dostarlimab clinical development program is also briefly discussed.

\section{Materials and methods Materials}

Critical reagents for this assay were as follows: a mouse anti-dostarlimab mAb (Precision Antibodies, Columbia, $\mathrm{MD})$ generated from clone \#6G10 at a concentration of $1.15 \mathrm{mg} / \mathrm{mL}$; dostarlimab (WuXi, Philadelphia, PA) at a concentration of $20.7 \mathrm{mg} / \mathrm{mL}$; biotinylated human PD-1 (ACRO Biosystems, Newark, DE) at a concentration of $200 \mu \mathrm{g} / \mathrm{mL}$; and SULFO-TAG-labeled human PD-L1 (CR0; Meso Scale Diagnostics [MSD], Rockville, MD) at a concentration of $2106 \mu \mathrm{g} / \mathrm{mL}$.

\section{Preparation of dostarlimab drug solution}

The initial stock solution of dostarlimab was diluted in LowCross-Buffer (Boca Scientific, Westwood, MA) to a concentration of $1608 \mathrm{ng} / \mathrm{mL}$, which is 6 times the desired final concentration $(268 \mathrm{ng} / \mathrm{mL})$. The prepared dostarlimab solution was aliquoted and stored at $-70^{\circ} \mathrm{C}$.

\section{Preparation of assay control samples}

The negative control (NC) was prepared by adding 250 $\mu \mathrm{g} / \mathrm{mL}$ dostarlimab (GSK, Waltham, MA) into pooled normal human serum (pNHS; Bio IVT, Westbury, NY) and freezing the solution for $12 \mathrm{~h}$. After thawing, dostarlimab was removed from the NC samples via the drug removal procedure (see below). The low-positive control (LPC) and high-positive control (HPC) were prepared by mixing dostarlimab and anti-dostarlimab NAb clone $6 \mathrm{G} 10$ in pNHS at $250 \mu \mathrm{g} / \mathrm{mL}$ and $500 \mathrm{ng} / \mathrm{mL}$, respectively, for the LPC and at $250 \mu \mathrm{g} / \mathrm{mL}$ and $2000 \mathrm{ng} / \mathrm{mL}$, respectively, for the HPC. The solution was then subjected to the same freeze-thaw-drug removal procedure as the NC. 
Once prepared, the NC, LPC, and HPC were stored at $70{ }^{\circ} \mathrm{C}$.

\section{Drug removal procedure}

Excess dostarlimab was removed from serum and control samples prior to performing the NAb detection assay. Biotinylated human PD-1 stock solution $(200 \mu \mathrm{g} /$ $\mathrm{mL}$ ) was prepared by dissolving $200 \mu \mathrm{g}$ of PD-1 in $1 \mathrm{~mL}$ of $\mathrm{dH}_{2} \mathrm{O}$. Magnetic streptavidin Dynabeads (Thermo Fisher Scientific, Waltham, MA) were prepared by transferring $140 \mu \mathrm{L}$ of streptavidin magnetic beads to a tube (approximately $50 \%$ of bead volume) where they were subsequently washed once using the magnetic stand with $0.5 \mathrm{~mL}$ of wash/bind buffer. To bind human PD-1 to the magnetic beads, $70 \mu \mathrm{L}(14 \mu \mathrm{g})$ of biotinylated PD-1 protein was added to the washed beads, and the mixture was incubated for 1 hour at ambient temperature while shaking at $1000 \mathrm{rpm}$. The PD-1 solution was then removed from the beads using the magnetic stand, and the beads were washed 3 times using the magnetic stand with 0.5 $\mathrm{mL}$ wash/bind buffer.

The positive control (PC) was prepared by adding dostarlimab and NAb to pNHS to achieve a final concentration of $250 \mu \mathrm{g} / \mathrm{mL}$ of dostarlimab and the desired concentration of NAb in $70 \mu \mathrm{L}$ of pNHS. Drug removal was accomplished by adding a one-tenth volume of $1 \mathrm{M}$ acetic acid to serum or control solutions and incubating at ambient temperature for $2 \mathrm{~h}$, resulting in a $\mathrm{pH}$ of 3.0 with no change of ADA affinity observed. A one-tenth volume of $1 \mathrm{M}$ Tris base was added to neutralize the samples.

The sample solution was immediately added to the prewashed PD-1-coated magnetic beads and incubated for 1 hour at ambient temperature while shaking at $1000 \mathrm{rpm}$. The samples were then separated from the streptavidin beads by magnetic separation and subsequently used in the NAb assay, or alternatively, stored at $-70{ }^{\circ} \mathrm{C}$.

\section{NAb detection assay}

The streptavidin-coated Gold SECTOR plates (MSD) were blocked with $1 \%$ bovine serum albumin (Gemini Bio, West Sacramento, CA) in phosphate-buffered saline (PBS) for 1 hour at ambient temperature with shaking at $450 \mathrm{rpm}$ and thoroughly washed (3 cycles on a plate washer with $0.05 \%$ Tween-20 in PBS). In a dilution plate, $20 \mu \mathrm{l}$ of prepared dostarlimab solution $(1608 \mathrm{ng} / \mathrm{mL})$ was added to $60 \mu \mathrm{L}$ of drug removal samples or controls, and the resulting solutions were incubated for 1 hour at ambient temperature with shaking at $450 \mathrm{rpm}$. A Master Mix solution of $12 \mathrm{nM}$ biotinylated PD-1 and $12 \mathrm{nM}$ SULFOTAG-labeled human PD-L1 was prepared in Low CrossBuffer. Then, $40 \mu \mathrm{L}$ of Master Mix was added to each well of the dilution plate, which was incubated for 1 hour at ambient temperature with shaking at $450 \mathrm{rpm}$ in the dark. The final drug concentration was $268 \mathrm{ng} / \mathrm{mL}$, which was selected based on the following: the linearity range for dostarlimab drug concentration to ECL signal was found to be $0-300 \mathrm{ng} / \mathrm{mL}$; the highest drug concentration in this range led to the lowest ECL signal in the test sample; and any ECL signal increase due to NAb interaction in the assay introduced a better signal to noise ratio. Therefore, approximately $90 \%$ of the $300 \mathrm{ng} / \mathrm{mL}$ (which was considered to be the high limit of quantification) was used to maintain the assay reproducibility. From each well of the dilution plate, $50 \mu \mathrm{L}$ was transferred to the washed streptavidin-coated plate and incubated for 1 hour at ambient temperature in the dark. Following incubation, the wells were washed with Tween-20 PBS, as described above, then $100 \mu \mathrm{L}$ of $2 \times$ Read Buffer (MSD) was added to each well. The plate was subsequently read on an MSD SECTOR Imager (MSD) to attain the electrochemiluminescence (ECL) signal.

\section{Statistical methods \\ Data description}

Sixty individual human cancer serum samples were obtained from the disease population, were divided into 4 groups with $30,30,40$, and 20 samples in groups A, B, C, and $\mathrm{D}$, respectively. The plate for group $\mathrm{D}$ also included 11 sensitivity samples. Each group was tested twice by 2 analysts across 3 days. Each analyst tested at least 1 set of sensitivity samples. The reported value for each test sample was the mean ECL response from duplicate wells on a plate. The NCs were tested 4 times on each plate, and the means of the duplicate wells were reported for a total of 32 ECL values. The LPC and HPC samples were tested twice on each plate for a total of 16 mean ECL values for each PC.

\section{Sources of variation}

All statistical analyses were completed using JMP Pro version 12.1.0 (JMP; Cary, NC), and methods used were consistent with recommendations by Shankar et al. (2008). Statistical outliers were determined using a quartile range outlier method. The statistical outliers in the upper and lower quartiles were defined as Q3 $+1.5 \times$ $(\mathrm{Q} 3-\mathrm{Q} 1)$ and $\mathrm{Q} 1-1.5 \times(\mathrm{Q} 3-\mathrm{Q} 1)$, where $\mathrm{Q} 3$ is the 75th percentile and Q1 is the 25th percentile. Outliers identified by these criteria were removed, and the model was re-fit. Normality of residual values was evaluated by the Shapiro-Wilk test (Mire-Sluis et al. 2004). In addition, the skewness coefficient was calculated as a relative measure of symmetry (Shapiro and Wilk 1965).

If skewness coefficient was less than -1 or greater than +1 , the distribution of the data was highly skewed; if skewness was between -1 and $-1 / 2$ or between $+1 / 2$ and +1 , the distribution was moderately skewed; and if 
skewness was between $-1 / 2$ and $+1 / 2$, the distribution was approximately symmetric. The parametric cut point estimates are typically recommended if normality distribution is confirmed (if $P>0.05$ ); however, if normality cannot be assumed, the parametric cut point estimate may be used if the data are not highly skewed (if skewness is between -1 and +1 ).

\section{NAb assay cut point}

Signal to noise $(S / N)$ ratio values were obtained by dividing the ECL signal value from each individual serum sample by the mean of the NC ECL values from the corresponding plate. After exclusion of analytic outliers from the set of NC values, the remaining $S / N$ ratio values were used for statistical analyses. Homogeneity of inter-plate sample variances across the 8 plates was investigated per Levene's test using $S / N$ ratio values (Levene 1960).

\section{Cut point factor determination}

Floating cut point factors (CPFs) for parametric and non-parametric screening were determined using the $S / N$ ratio values. A parametric method was used to calculate robust estimates of the mean and standard deviation (SD) of all $S / N$ ratio values. The parametric $1 \%$ error rate floating $\mathrm{CPF}$ was then determined by multiplying the $\mathrm{SD}$ value by the 99th quartile of the $t$-distribution (with degrees of freedom equal to the number of $S / N$ ratio values minus 1) and adding the product to the mean of $S / N$ ratio values. The non-parametric $1 \%$ error rate $\mathrm{CPF}$ was determined by calculating the empirical 99th percentile of $S / N$ ratio values.

\section{False-negative error rate}

The false-negative error rate, defined as the probability of observing an $S / N$ ratio less than the CPF in a known positive sample, was calculated based on the mean and SD of all LPC $S / N$ ratio values and a $t$-distribution with degrees of freedom equal to the number of ratio values minus 1 .

\section{Method validation}

Full method validation was completed for the aspects of intra- and inter-assay precision, sensitivity, selectivity, drug tolerance, interference by TSR-022, hook effect, assay robustness, and stabilities.

\section{Sensitivity}

Assay sensitivity was defined as the concentration of antidostarlimab NAbs spiked in pNHS with dostarlimab at $250 \mu \mathrm{g} / \mathrm{mL}$ followed by drug removal treatment, which consistently generated a signal above or equal to the applicable cut point. The overall sensitivity was equal to the average concentration of all plates $+t_{0.05, \mathrm{z} \text { (one side) }} \times$ $\mathrm{SD}$. Multiple levels of NAb PCs were prepared by spiking desired NAb concentrations that span the cut point into pNHS with dostarlimab at $250 \mu \mathrm{g} / \mathrm{mL}$. The sensitivity samples underwent the drug removal treatment prior to analysis. Six runs were performed by 2 analysts over 3 separate days.

\section{Assay precision}

Intra-assay precision was evaluated by running 6 independent validation samples for NCs, LPCs $(533.6 \mathrm{ng} /$ $\mathrm{mL})$, and HPCs $(2000 \mathrm{ng} / \mathrm{mL})$ on a single plate. The interassay precision was measured using the PCs and NCs in all accepted validation runs.

\section{Hook effect}

To determine if the assay demonstrated a hook effect, an ultrahigh concentration $(20,000 \mathrm{ng} / \mathrm{mL})$ of NAb was prepared in pNHS. Serial 2-fold dilutions of this sample in pNHS generated samples at 10,000, 5000, 2500, 1250, $625,312.5,156.25,78.13$, and $39.06 \mathrm{ng} / \mathrm{mL}$, which were evaluated as replicates.

\section{Selectivity}

Ten individual cancer serum samples, $52 \%$ hemolyzed normal serum samples and 5 lipemic normal serum samples $(450 \mu \mathrm{g} / \mathrm{dL})$ containing dostarlimab at $250 \mu \mathrm{g} / \mathrm{mL}$ (a) unspiked and (b) spiked with anti-dostarlimab NAb at the LPC level $(533.6 \mathrm{ng} / \mathrm{mL})$, underwent drug removal treatment and were tested.

\section{Drug interference}

Interference in the detection of LPC was evaluated by using the isotype IgG4 antibody TSR-022, which is an anti-TIM3 antibody. The results were considered acceptable if the TSR-022-spiked LPC sample values were within their respective ranges, which were established during this validation. Drug interference was tested using 3 sets of LPC and NC (a) unspiked and (b) spiked with TSR-022 at 1350, 450, and $150 \mu \mathrm{g} / \mathrm{mL}$. The drug removal treatment was applied to drug tolerance samples prior to analysis.

\section{Robustness}

The robustness of the assay was determined by varying the incubation time as described in Table S1. Plates were run concurrently and analyzed on the same day by a single analyst.

\section{Stability}

To assess the stability of the NAb, samples of HPC, LPC, and NC were stored in 3 different conditions: $4 \mathrm{~h}$ at ambient temperature, $24 \mathrm{~h}$ at $2-8{ }^{\circ} \mathrm{C}$, or subject to 6 freeze-thaw cycles. 


\section{System suitability}

The assay suitability criteria were established using the data from 15 of the 19 validation runs (robustness was not used to establish criteria).

\section{Results}

A competitive ligand-binding assay was designed to detect NAbs against dostarlimab. In brief, biotinylated human PD-1 captured on a streptavidin-coated plate binds with SULFO-TAG-labeled human PD-L1 to generate the ECL assay signal. Additionally, the intensity of the ECL signal is proportional to the binding amount of PD-1 and PD-L1 complex. When the constant amount of dostarlimab is added, it competitively binds to PD-1, which results in the inhibition of the assay signal from PD-1 and PD-L1 complex. In the presence of anti-dostarlimab NAb, dostarlimab can be neutralized and unable to bind with PD-1, which triggers the increase of assay signal corresponding to a NAb-positive result (Fig. 1).

In the study, samples from patients treated with dostarlimab may contain excess drug, potentially resulting in assay interference. Thus, the excess dostarlimab must be removed before the assay via the drug removal procedure (Fig. 2; see "Materials and methods" for the drug removal procedure). The drug removal efficiency was monitored through an enzyme-linked immunoassay (ELISA) assay with the validated range of 32.0$814 \mathrm{ng} / \mathrm{mL}$ (data on file). This process confirmed that

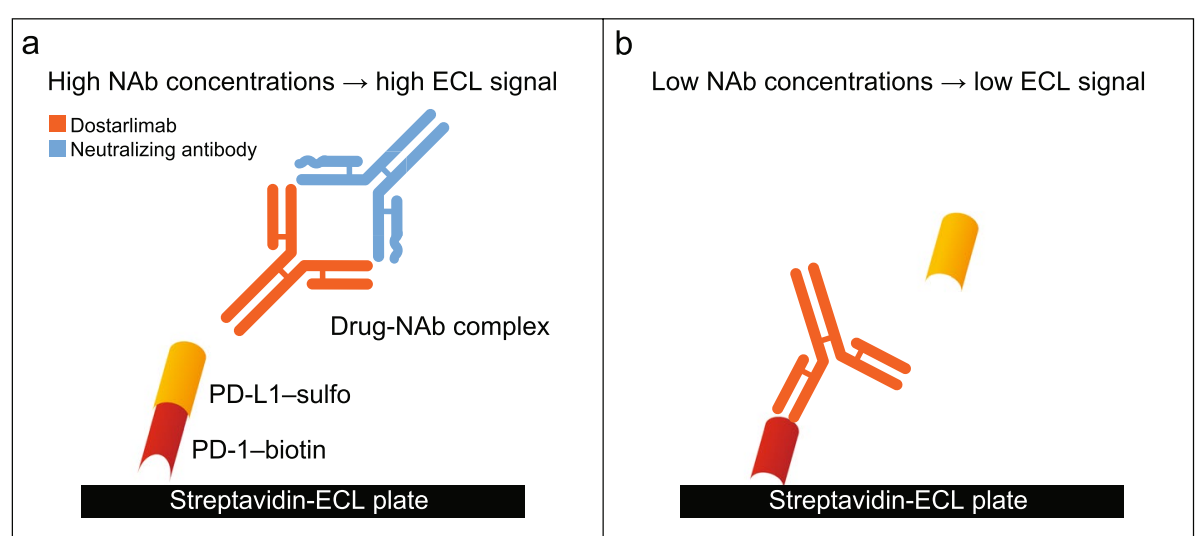

Fig. 1 NAb detection assay. A Complexes form between the fixed amount of added drug and NAb; the formation of these complexes allows SULFO-TAG-labeled PD-L1 to bind to biotinylated PD-1, which can bind to the streptavidin-ECL plate. B In the absence of NAb, the fixed amount of drug added to the solution binds to biotinylated PD-1 and prevents its interaction with PD-L1. SULFO-TAG-labeled PD-L1 bound to PD-1 is necessary to generate the ECL signal. ECL, electrochemiluminescence; NAb, neutralizing antibody; PD-1, programmed death 1; PD-L1, programmed death ligand 1

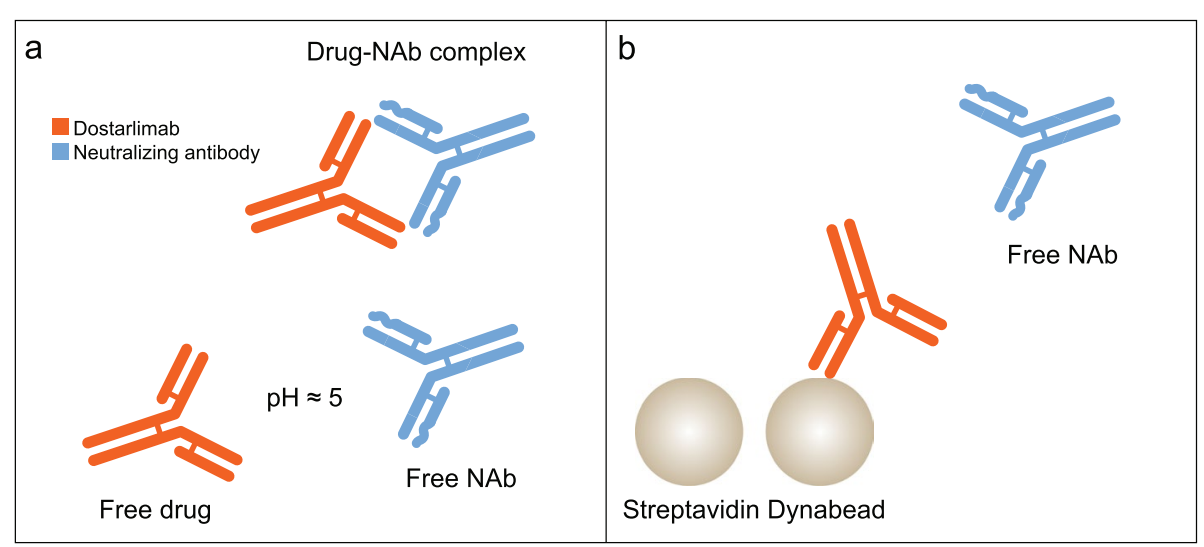

Fig. 2 Drug removal procedure. a Acidification of serum solution containing drug-NAb complexes with $1 \mathrm{M}$ acetic acid separates the drug from the drug-NAb complex. b Free drug is captured by PD-1-biotin that is bound to streptavidin magnetic Dynabeads, allowing the free NAb to be detected in the NAb detection assay. NAb, neutralizing antibody; PD-1, programmed death 1 
the processed samples had below quantification limit results in the ELISA assay, thus demonstrating that at least $250 \mu \mathrm{g} / \mathrm{mL}$ of the drug could be removed during the drug removal process. The drug removal step allows the assay to gain drug tolerance of at least $250 \mu \mathrm{g} / \mathrm{mL}$, which matches the level required for the 3-tier bridging ADA assay (data on file).

\section{Sources of variation Negative control}

Figure 3 illustrates the variation in NC ECL values listed across assay runs and plates. No NC values had a high percent coefficient of variation ( $\% \mathrm{CV}>20 \%)$, and no values were identified as statistical outliers, leaving all $32 \mathrm{NC}$ values in the final analysis. The Levene test for homogeneity of variances for runs and analysts showed that the variances of NC ECL values among runs and analysts were equal (Levene test, $P=0.1073$ ).

\section{$\mathrm{S} / \mathrm{N}$ ratio values}

Figure 4 provides scatter diagrams with box plots and variances of the serum $S / N$ ratio values versus the assay run and analyst from 60 cancer sera. The boxplot data indicated no statistically significant difference between runs and analysts. The Levene test of the $S / N$ ratio values showed that the variances were equal among runs and analysts (Levene test, $P=0.1006$ ).

\section{NAb assay cut point and CPF}

The NAb assay cut point (ACP) was defined as the level of assay response above or below which a sample is potentially positive or negative for the presence of NAbs. To evaluate the ACP and CPF, 60 individual human serum samples underwent the drug removal process and were screened for ACP factor determination along with control samples over 8 runs using 1 plate per run. Samples with $\%$ CV > 20\% were excluded from the data set and not included in the ACP calculations. Overall, $92.5 \%$ of data points were included

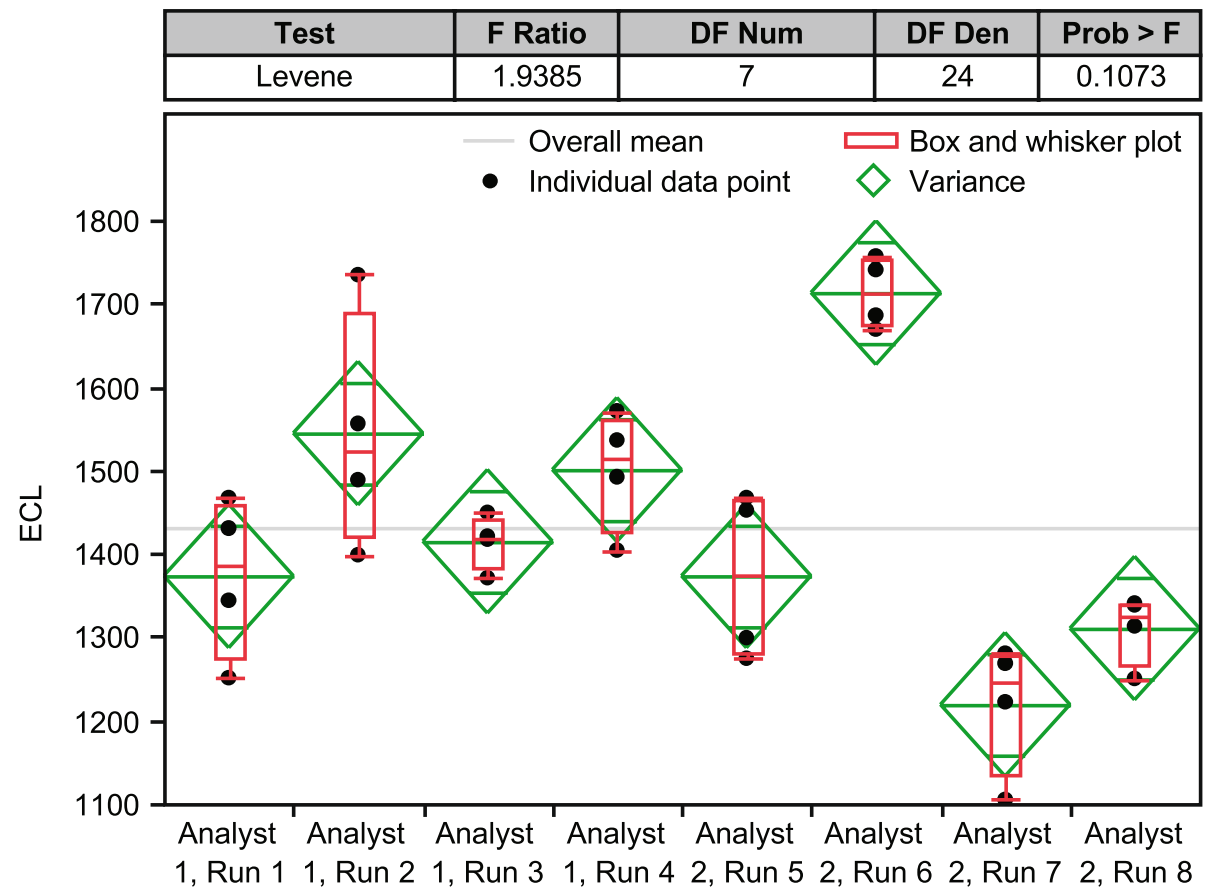

Fig. 3 Plot of negative control ECL values by run and analyst. Box and whisker plot of 25th and 75th percentiles, median, minimum, and maximum is presented. DF Den, denominator degree of freedom; DF Num, numerator degree of freedom; ECL, electrochemiluminescence; Prob, probability $S / N$, signal to noise

(See figure on next page.)

Fig. 4 Variation estimates by runs and analysts using human serum sample S/N ratio values, excluding high CV samples, voided samples, and outliers. Box and whisker plot of 25 th and 75 th percentiles, median, minimum, and maximum is presented. Analyst $1=(\mathrm{Q} 3+1.5 \times$ interquartile); analyst $2=(\mathrm{Q} 3+1.5 \times$ interquartile). $\mathbf{a}$ Estimation of variation between runs. $\mathbf{b}$ Estimation of variation between analysts. $\mathbf{c}$ Estimation of variation between analysts and runs. CV, coefficient of variation; DF Den, denominator degree of freedom; DF Num, numerator degree of freedom; ECL, electrochemiluminescence; Prob, probability $S / N$, signal to noise 


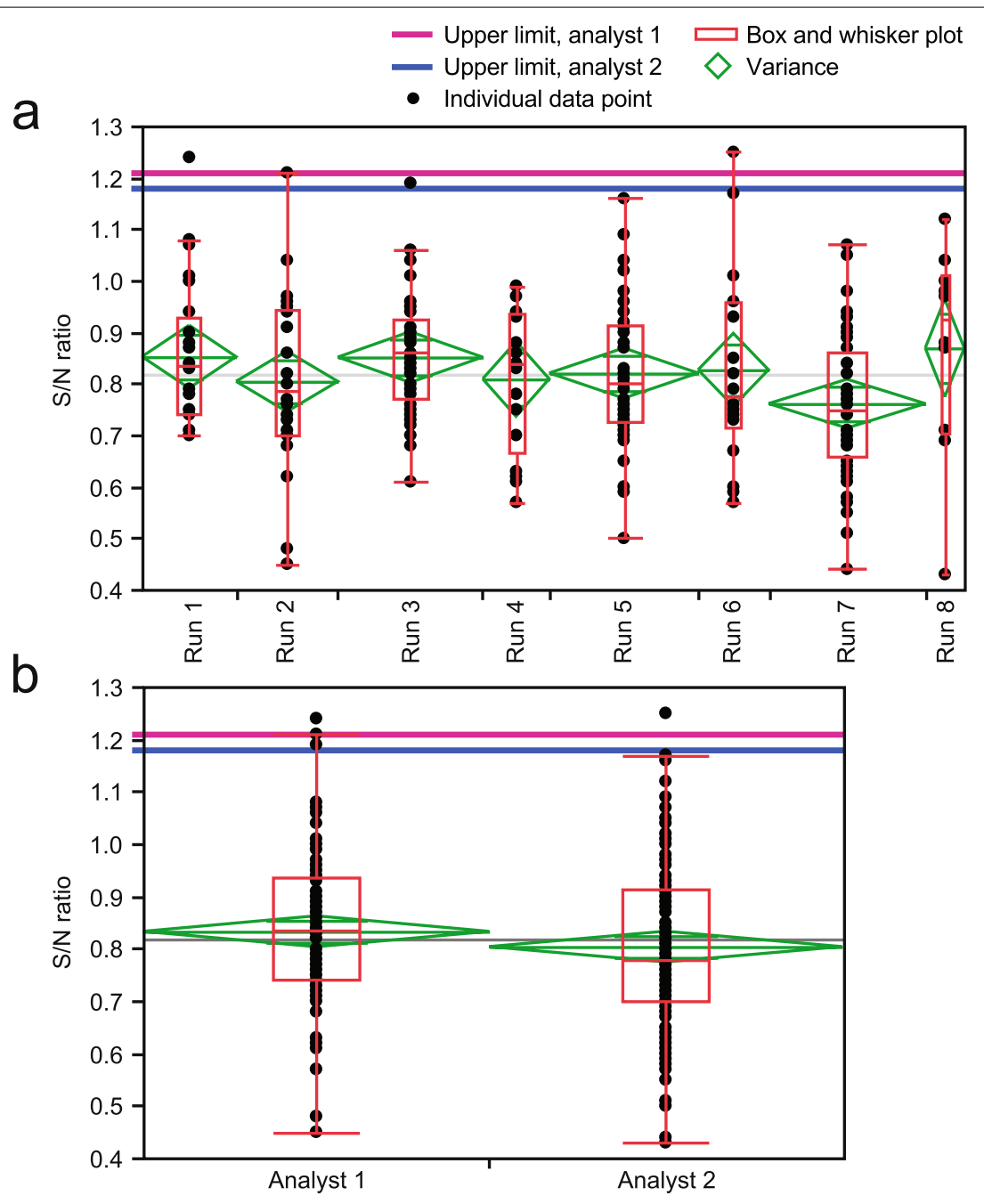

C

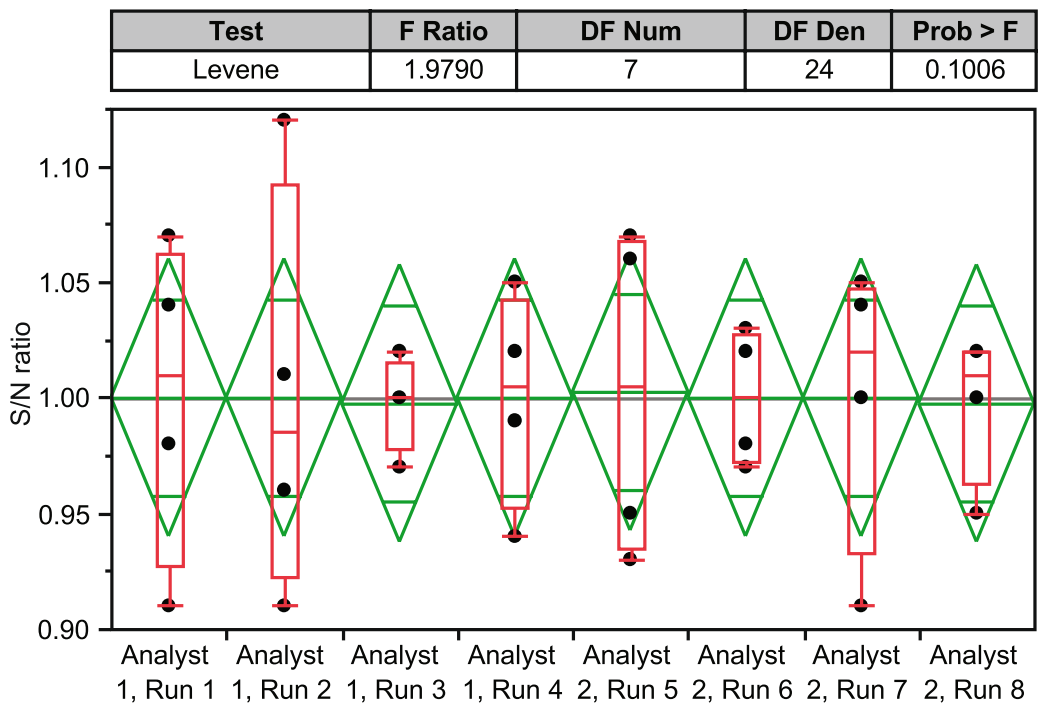

Fig. 4 (See legend on previous page.) 
in the calculation, and the overall mean of the rECL values (mean sample ECL value divided by mean plate NC ECL value) was determined. The distribution of the rECL values was found to be normal (the Shapiro-Wilk test, $P=0.3069$ ). The skewness coefficient was within acceptable limits $(P=$ $0.1955)$, suggesting the parametric estimate could be used. Figure 5 provides a scatter plot of the plate-specific ECL values from the human serum samples versus the ECL values of the NC on the corresponding plates. The linear relationship between sample ECL and NC ECL is shown with a slope of $0.92(P<0.0001)$, supporting the application of a floating CPF for screening test samples for the presence of dostarlimab NAbs.

After excluding the statistical outliers by box plot analysis (high limit $=\mathrm{Q} 3+1.5 \times[\mathrm{Q} 3-\mathrm{Q} 1]$ and low limit $=\mathrm{Q} 1-$ $1.5 \times$ [Q3 - Q1], where Q3 is the 75th percentile and Q1 is the 25 th percentile), the ACP was calculated by parametric analysis using the following equations:

$$
\mathrm{CPF}=\text { mean } S / N(\mathrm{rECL})+2.33 \times \mathrm{SD} \text {, }
$$
where 2.33 is the 99 th percentile of the normal distribution; $\mathrm{ACP}=\mathrm{CPF}$ $\times$ mean NC plate.

The CPF was found to be 1.18; thus, the plate-specific cut points were calculated using the following equation:

$$
\mathrm{ACP}=\text { mean NC plate } \times 1.18
$$

\section{False-negative error rate}

An estimate of the LPC false-negative error rate was determined based on the distribution of $S / N$ ratio values, where $S$ was the ECL for the LPC and $N$ was the mean of the ECL of the NC on the same plate. The probability of an $S / N$ ratio less than the parametric CPF of 1.18 was determined by calculating the probability of a $t$-distribution with 15 degrees of freedom having a $t$-value less than ([NAb CPF - mean of $S / N$ ratio values]/SD of $S / N$ ratio values). The false-negative error rate was computed to be $<0.1 \%$ with all the LPC (500 $\mathrm{ng} / \mathrm{mL}) S / N$ ratio values being above the 1.18 factor. The false-negative rate is a regulatory concern. With a defined CPF, the likelihood for samples with NAb concentrations as low as $500 \mathrm{ng} / \mathrm{mL}$ to be determined as negative is minimal.

\section{Assay validations Sensitivity (Table 1)}

Assay sensitivity was determined to be $476.5 \mathrm{ng} / \mathrm{mL}$ using the formula

Assay sensitivity $=339.3 \mathrm{ng} / \mathrm{mL}+(1.645 \times 83.4)=476.5 \mathrm{ng} / \mathrm{mL}$.

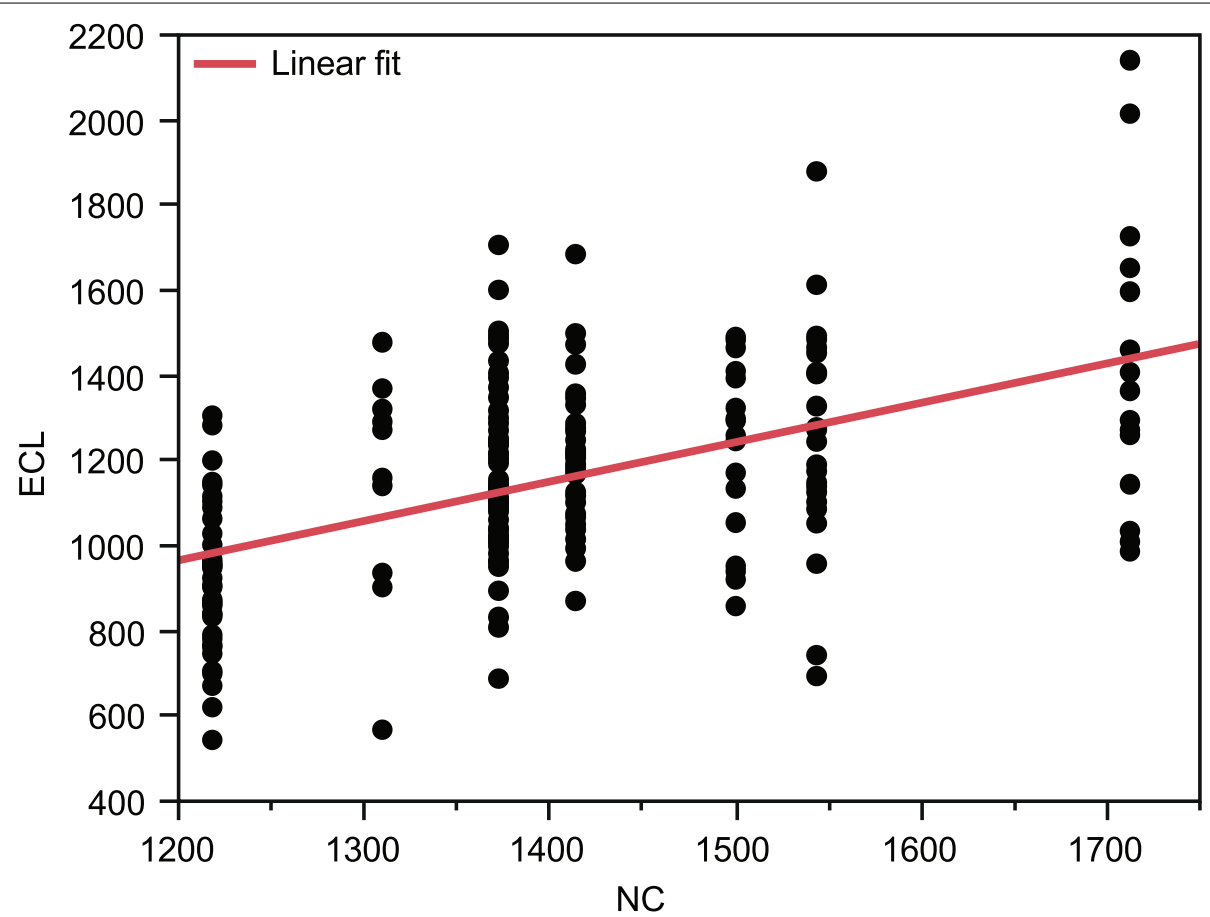

Fig. 5 Evaluation of human serum ECL values by corresponding negative control, excluding high CV samples, voided samples, and outliers. Linear fit: $E C L=-147.9245+0.9242683 \times N C$. ECL, electrochemiluminescence; NC, negative control 
Table 1 Assay sensitivity

\begin{tabular}{ll}
\hline Run ID & $\begin{array}{l}\text { CP corresponding } \\
\text { concentration }(\mathbf{n g} / \\
\mathbf{m L})^{\mathbf{a}}\end{array}$ \\
\hline Run 4 & 387.9 \\
Run 6 & 366.2 \\
Run 9 & 431.0 \\
Run 10 & 221.4 \\
Run 11 & 289.8 \\
Mean & 339.3 \\
SD & 83.4 \\
Sensitivity & $476.5^{\mathrm{b}}$ \\
\hline
\end{tabular}

$C P$ cut point, $S D$ standard deviation

${ }^{a}$ Regression model: 5PL (auto) with weighting factor of $1 / \mathrm{F}^{\wedge} 2$

${ }^{\mathrm{b}}$ Sensitivity was calculated using "average concentration $+t 0.05, Z$ (one side) $\times \mathrm{SD}^{\prime \prime}$

The established LPC $\left(\right.$ mean $\left.+\mathrm{t}_{0.01, \mathrm{z} \text { (one side) }} \times \mathrm{SD}=339.3 \mathrm{ng} / \mathrm{mL}+[2.33 \times 83.4]\right)$ was $533.6 \mathrm{ng} / \mathrm{mL}$ with a $1 \%$ false - negative rate.

\section{Assay precision}

Intra-assay precision was evaluated by running 6 independent validation samples for each level (NC, LPC, and $\mathrm{HPC}$ ) on a single plate. The $\% \mathrm{CV}$ of the intra-assay precision was 7.7, 7.5, and 8.1 for the HPC, LPC (500 ng/mL), and NC respectively (Table 2 ). The $\% \mathrm{CV}$ of the inter-assay precision was 19.0, 23.9, 14.8, and 8.0 for the HPC, LPC-1 $(500 \mathrm{ng} / \mathrm{mL}), \mathrm{LPC}-2(533.6 \mathrm{ng} / \mathrm{mL})$, and $\mathrm{NC}$, respectively (Table 3 ). Therefore, the intra-assay precision and interassay precision results met the specified criteria of \%CV $\leq 20 \%$ and $\% \mathrm{CV} \leq 25 \%$, respectively (Gupta et al. 2011).

\section{Hook effect}

Results in Table S2 show that the ECL signal of the NAb sample with the highest concentration $(20,000 \mathrm{ng} / \mathrm{mL})$ was above the cut point. Even though the signal levels

Table 2 Intra-assay precision

\begin{tabular}{llll}
\hline Quality control \# & $\begin{array}{l}\text { High-positive } \\
\text { control }\end{array}$ & $\begin{array}{l}\text { Low-positive } \\
\text { control }\end{array}$ & $\begin{array}{l}\text { Negative } \\
\text { control }\end{array}$ \\
\hline 1 & 9.4 & 7.4 & 1.0 \\
2 & 9.2 & 7.3 & 0.9 \\
3 & 9.8 & 7.7 & 0.9 \\
4 & 11.0 & 8.5 & 1.1 \\
5 & 9.2 & 7.7 & 1.0 \\
6 & 10.4 & 8.7 & 1.1 \\
Mean rECL & 9.8 & 7.9 & 1.0 \\
\%CV & 7.7 & 7.5 & 8.1 \\
\hline
\end{tabular}

$\% C V$ coefficient of variation, $E C L$ electrochemiluminescence, $r E C L$ mean sample $E C L$ value divided by mean plate negative control $E C L$ value
Table 3 Inter-assay precision

\begin{tabular}{ll}
\hline Overall inter-assay precision & \%CV \\
\hline High-positive control & 19.0 \\
Low-positive control 1 & 23.9 \\
Low-positive control 2 & 14.8 \\
Negative control & 8.0
\end{tabular}

$\% C V$ coefficient of variation

of NAb concentrations higher than $1250 \mathrm{ng} / \mathrm{mL}$ were slightly decreased, a large hook effect was not observed at higher NAb concentrations. Therefore, a positive signal would still be detected if the sample contained up to 20,000 ng/mL anti-dostarlimab NAbs.

\section{Selectivity}

Overall, 95\% of the spiked samples (human cancer serum, hemolyzed normal serum, and lipemic normal serum) had mean ECL values greater than the plate-specific cut point, and $90 \%$ of the unspiked samples had mean ECL values less than the plate-specific cut point (Table 4). Thus, the selectivity of the method met the acceptance criteria with all 3 serum types: cancer serum, hemolyzed normal serum, and lipemic normal serum.

\section{Drug interference}

As shown in Table 5, all TSR-022-spiked NC samples had ECL values below the cut point, and TSR-022-spiked LPC samples had ECL values above the cut point. Therefore, as long as the drug removal step is performed, even high levels of TSR-022 (up to $1350 \mu \mathrm{g} / \mathrm{mL}$ ) do not interfere with the signal for the LPC or NC.

\section{Assay robustness}

All robustness runs met the acceptance criteria (Table S3). Therefore, the assay was deemed robust with an incubation time ranging from 30 to 90 minutes per step: plate blocking, dostarlimab-NAb incubation, or sample incubation in MSD plate (Table S1).

\section{Stability}

To assess the stability of NAbs, samples of HPC, LPC, and NC were stored at 3 different conditions: $4 \mathrm{~h}$ at ambient temperature, $24 \mathrm{~h}$ at $2-8{ }^{\circ} \mathrm{C}$, or subject to 6 freezethaw cycles. All ECL signals of samples assessed for stability were within the established acceptance criteria (Table S4). Therefore, NAbs passed all the stability tests performed.

\section{System suitability criteria}

The assay suitability criteria were used as part of the run acceptance criteria to determine whether a run should 
Table 4 Assay selectivity

\begin{tabular}{|c|c|c|c|c|c|c|c|c|c|}
\hline \multirow[t]{2}{*}{ Sample type } & \multirow[t]{2}{*}{ Lot } & \multicolumn{4}{|c|}{ NAb unspiked } & \multicolumn{4}{|c|}{ NAb spiked at LPC } \\
\hline & & $\mathrm{ECL}$ & $\% C V$ & Plate CP & Condition & $\mathrm{ECL}$ & $\% \mathrm{CV}$ & Plate CP & Condition \\
\hline Cancer & 1 & 1113 & 2.9 & 1573 & $<\mathrm{CP}$ & 2745 & 7.2 & 1573 & $>C P$ \\
\hline Cancer & 2 & 1499 & 3.5 & 1573 & $<\mathrm{CP}$ & 2644 & 0.1 & 1573 & $>C P$ \\
\hline Cancer & 3 & 1917 & 0.8 & 1573 & $>C P$ & 2949 & 5.7 & 1573 & $>C P$ \\
\hline Cancer & 4 & 1352 & 2.1 & 1573 & $<\mathrm{CP}$ & 2820 & 4.9 & 1573 & $>C P$ \\
\hline Cancer & 5 & 1389 & 9.5 & 1573 & $<\mathrm{CP}$ & 2390 & 6.4 & 1573 & $>C P$ \\
\hline Cancer & 6 & 1269 & 0.8 & 1573 & $<\mathrm{CP}$ & 3382 & 4.9 & 1573 & $>C P$ \\
\hline Cancer & 7 & 1005 & 1.4 & 1573 & $<\mathrm{CP}$ & 2402 & 4.4 & 1573 & $>C P$ \\
\hline Cancer & 8 & 998 & 7.4 & 1573 & $<\mathrm{CP}$ & 2362 & 1.2 & 1573 & $>C P$ \\
\hline Cancer & 9 & 967 & 13.8 & 1573 & $<\mathrm{CP}$ & 1932 & 10.7 & 1573 & $>C P$ \\
\hline Cancer & 10 & 836 & 11.9 & 1573 & $<\mathrm{CP}$ & 2478 & 1.3 & 1573 & $>C P$ \\
\hline Hemolyzed & 1 & 1071 & 12.5 & 1573 & $<\mathrm{CP}$ & 2308 & 4.0 & 1573 & $>C P$ \\
\hline Hemolyzed & 2 & 1144 & 0.8 & 1573 & $<\mathrm{CP}$ & 2745 & 19.9 & 1573 & $>C P$ \\
\hline Hemolyzed & 3 & 893 & 9.0 & 1573 & $<\mathrm{CP}$ & 1717 & 3.4 & 1573 & $>C P$ \\
\hline Hemolyzed & 4 & 802 & 0.4 & 1573 & $<\mathrm{CP}$ & 2066 & 10.7 & 1573 & $>C P$ \\
\hline Hemolyzed & 5 & 553 & 2.0 & 1573 & $<\mathrm{CP}$ & 691 & 6.0 & 1573 & $<\mathrm{CP}$ \\
\hline Lipemic & 1 & 2561 & 6.3 & 1573 & $>C P$ & 3486 & 5.7 & 1573 & $>C P$ \\
\hline Lipemic & 2 & 1212 & 7.3 & 1573 & $<\mathrm{CP}$ & 2042 & 11.1 & 1573 & $>C P$ \\
\hline Lipemic & 3 & 813 & 1.4 & 1573 & $<\mathrm{CP}$ & 1652 & 5.2 & 1573 & $>C P$ \\
\hline Lipemic & 4 & 1525 & 6.0 & 1573 & $<\mathrm{CP}$ & 2758 & 1.0 & 1573 & $>C P$ \\
\hline Lipemic & 5 & 1010 & 0.8 & 1573 & $<\mathrm{CP}$ & 1916 & 10.6 & 1573 & $>C P$ \\
\hline
\end{tabular}

$C P$, cut point; \%CV, coefficient of variation; $E C L$, electrochemiluminescence readout; $L P C$, low-positive control; $N A b$, neutralizing antibody

Table 5 Drug interference

\begin{tabular}{|c|c|c|c|c|c|c|c|c|}
\hline \multirow[t]{2}{*}{ TSR-022 ( $\mu \mathrm{g} / \mathrm{mL})$} & \multicolumn{4}{|c|}{ NAb spiked at LPC level } & \multicolumn{4}{|c|}{ NAb unspiked } \\
\hline & $\mathrm{ECL}$ & $\% \mathrm{CV}$ & Plate CP & Condition & $\mathrm{ECL}$ & $\% \mathrm{CV}$ & Plate CP & Condition \\
\hline 1350 & 14676 & 7.4 & 2484.5 & $>C P$ & 2253 & 10.1 & 2484.5 & $<\mathrm{CP}$ \\
\hline 450 & 11298 & 12.7 & 2484.5 & $>C P$ & 2070 & 6.1 & 2484.5 & $<\mathrm{CP}$ \\
\hline 150 & 11444 & 8.8 & 2484.5 & $>C P$ & 1962 & 8.5 & 2484.5 & $<\mathrm{CP}$ \\
\hline 0 & 13930 & 8.1 & 2484.5 & $>C P$ & 2391 & 10.8 & 2484.5 & $<C P$ \\
\hline
\end{tabular}

$C P$ cut point, $\% C V$ coefficient of variation, $E C L$ electrochemiluminescence readout, $L P C$ low-positive control, $N A b$ neutralizing antibody

be rejected or accepted during validation stability testing and study sample analysis. The following system suitability criteria were obtained:

- NC range: rECL $0.76-1.24$

- LPC-1 range: rECL 1.82-11.01

- LPC-2 range: rECL 4.62-12.00

- HPC range: rECL 5.11-18.36

- $\mathrm{NC}<\mathrm{ACP}<\mathrm{LPC}<\mathrm{HPC}$

Analysis of all the validation runs showed that all QCs and NCs were within the acceptable range.

\section{Discussion}

Assay design

Initially, a cell-based assay was chosen to determine if it would meet the acceptance criteria to function as the NAb assay (Cong et al. 2015). This cell-based bioluminescent reporter assay was developed to quantify the $\mathrm{NAb}$ to the therapeutic mAbs for either anti-PD-1 or anti-PD-L1 that block the PD-1/PD-L1 interaction. PD-1 effector cells that express a nuclear factor of activated $\mathrm{T}$ cells (NFAT)-luciferase reporter and a human PD-1 receptor were engineered from Jurkat $\mathrm{T}$ cells (Cong et al. 2015). When the engineered Jurkat $T$ cells were 
cocultured with antigen-presenting cells that expressed $\mathrm{T}$ cell receptor (TCR) activator and PD-L1, activation of the NFAT pathway through TCR activator/TCR complex interaction would normally occur, leading to bioluminescence (Cong et al. 2015). However, engagement of PD-1 and PD-L1 on the cells would block the NFAT pathway and bioluminescence. Both anti-PD-1 and anti-PD-L1 mAbs were able to block the PD-1/PD-L1 interaction, resulting in the recovery of NFAT pathway signaling and bioluminescence (Cong et al. 2015). This assay, which can be used as a potency assay for anti-PD-1 drugs, was our starting point for NAb assay development. However, the assay was labor intensive, had low tolerance for serum, and lacked sensitivity (data on file), which did not allow the cell-based reporter assay to move forward as a validated anti-dostarlimab NAb assay.

The current trend for choosing a NAb assay format includes using the therapeutic MOA as the driving selection criterion and, in addition, relies on both assay performance characteristics and the risk of immunogenicity to the patient (Wu et al. 2016). When considering the MOA of the drug, both drug-target interaction and the structural characteristics need to be examined. Dostarlimab is an antagonistic $\mathrm{mAb}$ that directly blocks the function of the extracellular domains of PD-1, which are responsible for the interaction with PD-L1 and signal transduction to the intra-cellular domains. Since dostarlimab blocks the specific PD-1/PD-L1 interaction by binding to the PD-1 receptor, instead of binding at another site to exert its effect, a non-cell-based NAb assay is adequate for NAb detection (Wu et al. 2016). Two other anti-PD-1 mAbs, nivolumab and pembrolizumab, also directly block the interaction of PD-1 with its ligands. Currently, noncell-based assays are used for the detection of NAbs to nivolumab and pembrolizumab (van Vugt et al. 2019; Enrico et al. 2020).

\section{Assay performance and clinical relevance}

A cell-based assay was evaluated before the white paper on assay format assessment was published (Wu et al. 2016). Extensive optimization of the cell-based assay, including PD-L1 cell plating density, recovery of PD-1/PD-L1 co-culture or induction time, human serum concentration, cell culture plate type, luciferase substrate volume and reaction time, and plate reader setting, were completed to improve the assay sensitivity. Under optimal assay conditions, the sensitivity in the presence of $0.4 \mu \mathrm{g} / \mathrm{mL}$ of the drug was determined to be $12 \mu \mathrm{g} / \mathrm{mL}$ by screening 50 lots of human cancer serum. Since this was far from the USA Food and Drug Administration recommended assay sensitivity of $100 \mathrm{ng} / \mathrm{mL}$, it was concluded that this assay was highly insensitive and was not serviceable for NAb detection. Furthermore, unlike most other cell-based assays, which require only 1 type of cell line, the PD-1/ PD-L1 blockade bioassay required culturing, maintenance, and preparation of 2 independent cell lines, followed by co-culturing in an appropriate ratio to mimic PD-1/PD-L1 function in vivo. Undoubtedly, this would potentially create more uncertainty leading to less sensitive and less reproducible results.

The drug tolerance of this NAb assay was at least 250 $\mu \mathrm{g} / \mathrm{mL}$ with the implementation of the drug removal process. This level of tolerance is based on the exposure level observed from the recommended therapeutic dose (4 doses of $500 \mathrm{mg}$ Q3W followed by $1000 \mathrm{mg}$ Q6W afterwards) established in the GARNET study. The exposure evaluation was done at cycles $1,4,5,8$, and 12 , and only pre-dose samples were used for the immunogenicity evaluation. During the treatment process, the highest observed concentration, which was confirmed ADA-positive through 3-tier ADA assays, was lower than $250 \mu \mathrm{g} / \mathrm{mL}$. The drug tolerance of this assay allows all samples to be evaluated with the low inconclusive rate. In the NAb assay validation, the cut point was determined using 60 individual human serum samples with the CPF of 1.18 . The selection of the 60 cancer sera was balanced with respect to age, sex, and cancer types. This CPF can be applied to different indications without bias. The GARNET study includes multiple patient populations, and in its NAb evaluation, it is unclear whether this pre-study cut point is suitable for the population being studied (ClinicalTrials.gov 2016). Statistical determination of validation and instudy immunogenicity cut points were performed by the same statistician at Frontage Laboratory (Exton, PA) using the same procedures. An in-study cut point was determined statistically. $\mathrm{S} / \mathrm{N}$ ratio responses (also called $\mathrm{rECL}$ ) from 40 individual pre-dose baseline serum samples from the GARNET study that were negative in the $\mathrm{NAb}$ assay were used for the cut point analysis. Out of the $40 \mathrm{~S} / \mathrm{N}$ ratios, no outliers were identified, and all values were included in cut point determination. The normality of the $40 \mathrm{~S} / \mathrm{N}$ ratio responses was evaluated using the Shapiro-Wilk test, and the test gave a $P$ value $=0.0754$, which is more than $0.01(1 \%)$, indicating that the data set of $40 \mathrm{~S} / \mathrm{N}$ ratio values was considered to be normally distributed.

The in-study parametric CPF for the NAb was calculated as 1.35 at a $1 \%$ false-positive error rate. In the original NAb sample analysis based on the method validation CPF of 1.18 at the $1 \%$ false-positive error rate for the GARNET study, 64 of 135 confirmed ADA-positive samples were determined to be NAb positive. Upon retrospective application of the higher in-study cut point, 18 of the 64 samples switched from NAb positive 
to NAb negative. Based on the 3-tier ADA assays, 13 patients had treatment-emergent ADAs. Of these 13 patients, 7 patients were confirmed as NAb positive and the other 6 were NAb negative using both the validation cut point and the in-study cut point. Use of the higher in-study NAb cut point did not change the number of patients with treatment-emergent ADAs who were positive for NAbs, and thus would have no effect on the overall interpretation of the immunogenicity findings for this study using the tiered approach of 3-tier ADA assays and this NAb assay. Furthermore, method sensitivity and drug tolerance should not be greatly impacted by the lot of serum samples used for the establishment of cut point(s). The 60 lots of serum samples were selected to balance for cancer type, age, and sex. In addition, only 40 pre-dose samples from the study were used to calculate the in-study cut point, which was not optimal. Method sensitivity and drug tolerance should be evaluated by spiking in the pooled pre-dose samples of the study. This was not required here since the validation cut point can be further used due to limited impact as demonstrated in the above analysis results. With the limited positive ADA data in the GARNET study, no clinically relevant impact was observed from PK, safety, or efficacy, which is consistent with the observation that in currently approved anti-PD-1 mAbs, the presence of ADAs and NAbs does not correlate with an impact on pharmacokinetics, pharmacodynamics, safety, or efficacy (Davda et al. 2019; Enrico et al. 2020).

\section{Conclusions}

Because the clinical implications of ADAs and NAbs against dostarlimab remain unclear, both ADAs and NAbs to dostarlimab should be accurately detected using an established assay, such as that described here (Enrico et al. 2020). As has been demonstrated with other anti-PD-1 mAbs, a validated non-cell-based competitive ligand-binding assay is appropriate for detecting NAbs against dostarlimab with suitable precision, sensitivity, and selectivity (Enrico et al. 2020; Center for Drug Evaluation and Research 2017). Detection of ADAs, especially NAbs, is extremely important for the evaluation of efficacy and safety of biologic therapeutics. NAbs act by neutralizing biologic therapeutics; therefore, their presence may diminish drug efficacy. To this end, we have established an assay that can detect NAbs against dostarlimab, a humanized anti-PD-1 antibody. We have determined the CPF for the detection of neutralizing anti-dostarlimab antibodies in human serum at a $1 \%$ false-positive rate. The assay's precision, sensitivity, hook effect, selectivity, robustness, and drug interference were tested and found to be acceptable. The performance of the assay was independent of runs and analysts. With the system's suitability, stability, and drug tolerance, which covers the drug concentration range for the therapeutic dose established, this assay has been used to evaluate NAbs to support the clinical trials that help guide the development of dostarlimab.

\begin{abstract}
Abbreviations
ACP: Assay cut point; ADAs: Anti-drug antibodies; CPFs: Cut point factors; CV: Coefficient of variation; dMMR: Mismatch repair-deficient; ECL: Electrochemiluminescence; ELISA: Enzyme-linked immunoassay; HPC: High-positive control; LPC: Low-positive control; mAb: Monoclonal antibody; MOA: Mechanism of action; MSD: Meso Scale Diagnostics; NAbs: Neutralizing antibodies; NC: Negative control; NFAT: Nuclear factor of activated T cells; PBS: Phosphate-buffered saline; PD-1: Programmed death 1; PD-L1: Programmed death ligand 1; PK:

Pharmacokinetic; pNHS: Pooled normal human serum; SD: Standard deviation; $S / N$ : Signal to noise; TCR: T cell receptor.
\end{abstract}

\section{Supplementary Information}

The online version contains supplementary material available at https://doi. org/10.1186/s41120-021-00039-w.

Additional file 1: Table S1. Validation of Assay's Robustness. Table S2. Hook Effect. Table S3. Assay's Robustness. Table S4. Assay Stability

\section{Acknowledgements}

Medical writing and editorial assistance, funded by GlaxoSmithKline (Waltham, Massachusetts) and coordinated by Hasan Jamal, MSc, of GlaxoSmithKline, were provided by Shannon Morgan-Pelosi, PhD, and Jennifer Robertson, PhD, of Ashfield MedComms, an Ashfield Health company (Middletown, CT, USA)

\section{Authors' contributions}

Allauthors provided substantial contributions to the conception or design of thework, or the acquisition, analysis, or interpretation of data for the work. Allauthors drafted the work, revised it critically for important intellectualcontent, and provided final approval of the version to be published. Allauthors had access to full data and analyses presented in this manuscript.

Authors' information

Not applicable

\section{Funding \\ Funding for this study was provided by GlaxoSmithKline (NCT02715284) Trademarks are owned by or licensed to the GSK group of companies.}

\section{Availability of data and materials}

GSK makes available anonymized individual participant data and associated documents from interventional clinical studies which evaluate medicines, upon approval of proposals submitted to www.clinicalstudydatarequest.com. To access data for other types of GSK sponsored research, for study documents without patient-level data, and for clinical studies not listed, please submit an inquiry via the website.

\section{Declarations}

\section{Competing interests}

Xiaolong Tom Zhang has nothing to disclose. Hong Chen has nothing to disclose. Weiping Shao has nothing to disclose. Zhongping John Lin has nothing to disclose. Murad Melhem is an employee of GlaxoSmithKline. Sharon Lu is an employee of Scholar Rock and was affiliated with GlaxoSmithKline at the time of the study analyses. 


\section{Author details}

${ }^{1}$ WuXi AppTec, 288 Fute Zhong Road, Waigaoqiao Free Trade Zone, Shanghai 200131, China. ${ }^{2}$ Alliance Pharma, 17 Lee Boulevard, Malvern, PA 19355, USA. ${ }^{3}$ Frontage Laboratories, 700 Pennsylvania Drive, Exton, PA 19341, USA. ${ }^{4}$ GlaxoSmithKline, 1000 Winter Street, Waltham, MA 02451, USA.

Received: 4 June 2021 Accepted: 13 October 2021

Published online: 11 November 2021

\section{References}

Ahn BC, Pyo KH, Xin CF, Jung D, Shim HS, Lee CY et al (2019) Comprehensive analysis of the characteristics and treatment outcomes of patients with non-small cell lung cancer treated with anti-PD-1 therapy in real-world practice. J Cancer Res Clin Oncol 145(6):1613-1623

Andre T, Berton D, Curigliano G, Ellard S, Perez JMT, Arkenau HT et al (2021) Safety and efficacy of anti-PD-1 antibody dostarlimab in patients with mismatch repair-deficient (dMMR) solid cancer: results from the GARNET study. J Clin Oncol 39(suppl 3):abstr 9

Ansell SM, Lesokhin AM, Borrello I, Halwani A, Scott EC, Gutierrez M et al (2015) PD-1 blockade with nivolumab in relapsed or refractory Hodgkin's lymphoma. N Engl J Med 37(4):311-319

Antonia SJ, Villegas A, Daniel D, Vicente D, Murakami S, Hui R, PACIFIC Investigators et al (2017) Durvalumab after chemoradiotherapy in stage III non-small-cell lung cancer. N Engl J Med 377(20):1919-1929

Apolo AB, Ellerton JA, Infante JR, Agrawal M, Gordon MS, Aljumaily R et al (2017) Updated efficacy and safety of avelumab in metastatic urothelial carcinoma ( $\mathrm{UUC}$ ): pooled analysis from 2 cohorts of the phase $1 \mathrm{~b}$ Javelin solid tumor study. J Clin Oncol 35(15 suppl):4528

Bloem K, Hernández-Breijo B, Martínez-Feito A, Rispens T (2017) Immunogenicity of therapeutic antibodies: monitoring antidrug antibodies in a clinical context. Ther Drug Monit 39(4):327-332

Center for Drug Evaluation and Research (2017) Approval package for: application number 1255140rig1s014. https://www.accessdata.fda. gov/drugsatfda_docs/nda/2019/1255140rig1s014.pdf. Accessed 4 Feb 2021.

ClinicalTrials.gov (2016) Study of TSR-042, an anti-programmed cell death-1 receptor (PD-1) monoclonal antibody, in participants with advanced solid tumors (GARNET): NCT02715284. https://clinicaltrials.gov/ct2/show/ NCT02715284. Accessed 6 May 2020. https://clinicaltrials.gov/ct2/show/ NCT02715284?term $=02715284 \& d r a w=2 \&$ rank $=1$

Cong M, Cheng Z, Karassina N, Grailer J, Hartnett J, Cosby N, Fan F et al (2015) Advertorial: novel bioassay to assess PD-1/PD-L1 therapeutic antibodies in development for immunotherapy. Genet Eng News 35(10) https:// www.genengnews.com/magazine/247/advertorial-novel-bioassay-toassess-pd-1-pd-11-therapeutic-antibodies-in-development-for-immun otherapy/. Accessed 14 Aug 2020

Davda J, Declerck P, Hu-Lieskovan S, Hickling TP, Jacobs IA, Chou J et al (2019) Immunogenicity of immunomodulatory, antibody-based, oncology therapeutics. J Immunother Cancer 7:105

El-Khoueiry AB, Sangro B, Yau T, Crocenzi TS, Kudo M, Hsu C et al (2017) Nivolumab in patients with advanced hepatocellular carcinoma (CheckMate 040): an open-label, non-comparative, phase 1/2 dose escalation and expansion trial. Lancet. 389(10088):2492-2502

Enrico D, Paci A, Chaput N, Karamouza E, Besse B (2020) Antidrug antibodies against immune checkpoint blockers: impairment of drug efficacy or indication of immune activation? Clin Cancer Res 26(4):787-792

European Medicines Agency (2021) Jemperli. https://www.ema.europa.eu/en/ medicines/human/EPAR/jemperli. Accessed 24 May 2020

Fehrenbacher L, Spira A, Ballinger M, Kowanetz M, Vansteenkiste J, Mazieres J, POPLAR Study Group et al (2016) Atezolizumab versus docetaxel for patients with previously treated non-small-cell lung cancer (POPLAR): a multicentre, open-label, phase 2 randomised controlled trial. Lancet. 387(10030):1837-1846

Garcês S, Demengeot J (2018) The immunogenicity of biologic therapies. Curr Probl Dermatol 53:37-48

Gong J, Chehrazi-Raffle A, Reddi S, Salgia R (2018) Development of PD-1 and PD-L1 inhibitors as a form of cancer immunotherapy: a comprehensive review of registration trials and future considerations. J Immunother Cancer 6(1):8

Gunn GR, Sealey DCF, Jamali F, Meibohm B, Ghosh S, Shankar G (2016) From the bench to clinical practice: understanding the challenges and uncertainties in immunogenicity testing for biopharmaceuticals. Clin Exp Immunol 184(2):137-146

Gupta S, Devanarayan V, Finco D, Gunn GR III, Kirshner S, Richards S et al (2011) Recommendations for the validation of cell-based assays used for the detection of neutralizing antibody immune responses elicited against biological therapeutics. J Pharm Biomed Anal 55(5):878-888

Jolicoeur P, Tacey RL (2012) Development and validation of cell-based assays for the detection of neutralizing antibodies to drug products: a practica approach. Bioanalysis. 4(24):2959-2970

Kaufman HL, Russell J, Hamid O, Bhatia S, Terheyden P, D’Angelo SP et al (2016) Avelumab in patients with chemotherapy-refractory metastatic Merkel cell carcinoma: a multicentre, single-group, open-label, phase 2 trial. Lancet Oncol 17(10):1374-1385

Khozin S, Miksad RA, Adami J, Boyd M, Brown NR, Gossai A et al (2019) Realworld progression, treatment, and survival outcomes during rapid adoption of immunotherapy for advanced non-small cell lung cancer. Cancer. 125(22):4019-4032

Krishna M, Nadler S (2016) Immunogenicity to biotherapeutics - the role of anti-drug immune complexes. Front Immunol 7:21

Levene H (1960) Robust tests for the equality of variances. In: Oaklin I (ed) Contributions to probability and statistics. Stanford University Press, Palo Alto, pp 278-292

Liao K, Sikkema D, Wang C, Chen K, DeWall S, Lee TN (2012) Development and validation of a cell-based SEAP reporter assay for the detection of neutralizing antibodies against an anti-IL-13 therapeutic antibody. J Immunol Methods 375(1-2):258-263

Mire-Sluis AR, Barrett YC, Devanarayan V, Koren E, Liu H, Maia M et al (2004) Recommendations for the design and optimization of immunoassays used in the detection of host antibodies against biotechnology products. J Immunol Methods 289:1-16

Oaknin A, Ellard SL, Leath C III, Moreno V, Kristeleit R, Guo W et al (2018) Preliminary safety, efficacy, and PK/PD characterization from GARNET, a phase 1 clinical trial of the anti-PD-1 monoclonal antibody, TSR-042, in patients (pts) with recurrent or advanced MSI-H endometrial cancer. Ann Oncol 29(suppl8):viii334

Pineda C, Hernandez GC, Jacobs IA, Alvarez DF, Carini C (2016) Assessing the immunogenicity of biopharmaceuticals. BioDrugs. 30:195-206

Robert C, Ribas A, Wolchok JD, Hodi FS, Hamid O, Kefford R et al (2014) Anti-programmed-death-receptor-1 treatment with pembrolizumab in ipilimumab-refractory advanced melanoma: a randomised dose-comparison cohort of a phase 1 trial. Lancet. 384(9948):1109-1117

Seiwert TY, Burtness B, Mehra R, Weiss J, Berger R, Eder JP et al (2016) Safety and clinical activity of pembrolizumab for treatment of recurrent or metastatic squamous cell carcinoma of the head and neck (KEYNOTE-012): an open-label, multicentre, phase $1 \mathrm{~b}$ trial. Lancet Oncol 17(7):956-965

Shankar G, Devanarayan V, Amaravadi L, Barrett YC, Bowsher R, Finco-Kent D et al (2008) Recommendations for the validation of immunoassays used for detection of host antibodies against biotechnology products. J Pharm Biomed Anal 48(5):1267-1281

Shapiro SS, Wilk MB (1965) Analysis of variance test for normality (complete samples). Biometrika. 52:591-611

US Food and Drug Administration. (2021a) FDA grants accelerated approval to dostarlimab-gxly for dMMR endometrial cancer. https://www.fda. gov/drugs/drug-approvals-and-databases/fda-grants-acceleratedapproval-dostarlimab-gxly-dmmr-endometrial-cancer. Accessed 24 May 2021.

US Food and Drug Administration. (2021b) FDA grants accelerated approval to dostarlimab-gxly for dMMR advanced solid tumors. https://www.fda.gov/ drugs/resources-information-approved-drugs/fda-grants-acceleratedapproval-dostarlimab-gxly-dmmr-advanced-solid-tumors. Accessed 16 Sept 2021.

van Vugt MJH, Stone JA, De Greef RHJMM, Snyder ES, Lipka L, Turner DC et al (2019) Immunogenicity of pembrolizumab in patients with advanced tumors. J Immunother Cancer 7(1):212 
Weber JS, D'Angelo SP, Minor D, Hodi FS, Gutzmer R, Neyns B et al (2015) Nivolumab versus chemotherapy in patients with advanced melanoma who progressed after anti-CTLA-4 treatment (CheckMate 037): a randomised, controlled, open-label, phase 3 trial. Lancet Oncol 16(4):375-384

Wu B, Chung S, Jiang XR, McNally J, Pedras-Vasconcelos J, Pillutla R et al (2016) Strategies to determine assay format for the assessment of neutralizing antibody responses to biotherapeutics. AAPS J 18(6):1335-1350
Wu Y, Akhgar A, Li J, Yu B, Chen C, Lee N et al (2018) Selection of a ligandbinding neutralizing antibody assay for benralizumab: comparison with an antibody-dependent cell-mediated cytotoxicity (ADCC) cell-based assay. AAPS. 20(3):49

\section{Publisher's Note}

Springer Nature remains neutral with regard to jurisdictional claims in published maps and institutional affiliations.

\section{Submit your manuscript to a SpringerOpen ${ }^{\circ}$ journal and benefit from:}

- Convenient online submission

- Rigorous peer review

- Open access: articles freely available online

- High visibility within the field

- Retaining the copyright to your article

Submit your next manuscript at $\boldsymbol{\nabla}$ springeropen.com 